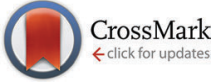

Cite this: Soft Matter, 2015, 11,9385

Received 19th June 2015, Accepted 25th September 2015

DOI: $10.1039 / \mathrm{c} 5 \mathrm{sm} 01520 \mathrm{k}$

www.rsc.org/softmatter

\title{
Dynamical heterogeneities and defects in two-dimensional soft colloidal crystals
}

\author{
B. van der Meer, ${ }^{\star a}$ W. Qi, ${ }^{a}$ J. Sprakel, ${ }^{b}$ L. Filion ${ }^{a}$ and M. Dijkstra*a
}

\begin{abstract}
In this paper we study a two-dimensional system of charged colloidal particles using Brownian dynamics simulations. We determine the phase diagram and investigate the dynamics of this system in the density regime where hexatic and solid phases are stable. We find that the dynamics in these phases is heterogeneous by means of the spontaneous formation and diffusion of highly mobile defects. We identify two key mechanisms associated with the areas of high mobility. The first mechanism involves the highly cooperative motion of a closed loop of particles which shift coherently along the loop until each particle has replaced the position of its predecessor in the chain. The second mechanism involves the spontaneous creation of vacancy-interstitial pairs which diffuse within the hexatic and solid phases. We further explore quantitatively the properties of the open-ended and closed rearrangement strings and find that in the crystal phase the string-size distribution can be approximately matched with a simple, random walk description of vacancies and interstitials on a lattice.
\end{abstract}

\section{Introduction}

Particles in a crystal are typically orders of magnitude less mobile than those in a fluid and usually diffuse via the motion of defects, such as vacancies and interstitials. Understanding the motion of these defects is key to understanding transport processes in crystals, including particle hopping and other local rearrangements, ${ }^{1-9}$ as well as mechanical instabilities, such as creep, fracture and yielding..$^{10-13}$

Simulation studies of two-dimensional systems have demonstrated the existence of strongly heterogeneous dynamics in both crystal and hexatic phases. ${ }^{14-16}$ In these systems, the dynamics becomes heterogeneous both in space and time: clusters of particles that rearrange cooperatively move in an intermittent manner at different rates. To date, studies of these dynamical heterogeneities have mainly addressed the dynamics on the ensemble level, i.e. in terms of time correlation functions. ${ }^{14,15}$ However, a detailed microscopic description of the cooperative motion of individual particles, and the defect structures associated with particle hopping events, is lacking. Therefore, the microscopic origin of the heterogeneous dynamics in colloidal crystals remains an open topic for investigation.

In a recent publication ${ }^{17}$ we examined, both in experiments and simulations, the heterogeneous dynamics associated with

\footnotetext{
${ }^{a}$ Soft Condensed Matter, Debye Institute for Nanomaterials Science, Utrecht University, Princetonplein 5, 3584 CC Utrecht, The Netherlands. E-mail: B.vanderMeer@uu.nl, m.dijkstra@uu.nl

${ }^{b}$ Physical Chemistry and Soft Matter, Wageningen University, Dreijenplein 6, 6703 HB Wageningen, The Netherlands
}

a local mechanical perturbation by means of optical tweezing. In particular, we perturbed a colloidal crystal by moving a single particle out of its equilibrium position, and explored the response of the system to this external perturbation. We observed that the system relaxed by two main mechanisms, namely the cooperative motion of closed loops of particles, and the formation and motion of dissociated vacancy-interstitial pairs. A preliminary investigation indicated that thermal stresses in the unperturbed system relax via the same cooperative motions. Here we return to this system and perform an in-depth analysis of the heterogeneities observed in this system. To this end we determine the phase diagram, i.e. the area fractions associated with the fluid to hexatic and hexatic to solid phase transitions. Using correlation functions, we determine the time scales for the dynamical heterogeneities, and quantitatively examine the hopping mechanisms responsible for diffusion in these systems.

\section{Methods}

In this paper, we consider a two-dimensional system of $N$ charged spherical particles with diameter $\sigma$ in an area $A$. The interaction between the particles is modelled using a Yukawa potential given by

$$
\beta U\left(r_{i j}\right)=\beta U_{0} \frac{\exp \left(-\kappa\left(r_{i j}-\sigma\right)\right)}{r_{i j} / \sigma},
$$

where $\beta=1 / k_{\mathrm{B}} T$ with $k_{\mathrm{B}}$ Boltzmann's constant and $T$ the temperature, and $r_{i j}=\left|\mathbf{r}_{i}-\mathbf{r}_{j}\right|$ is the center-of-mass distance between particle $i$ and $j$, with $\mathbf{r}_{i}$ the position of particle $i$. In all 
our simulations, we set the Debye screening length to $\kappa \sigma=2.25$ and the contact value to $\beta U_{0}=235$ in order to match a recent experimental realization of this system, studied in ref. 17.

This system displays two phase transitions as a function of the area fraction $\phi=\frac{\pi \sigma^{2} N}{4 A}$, namely a fluid to hexatic phase transition and a hexatic to hexagonal crystal transition. To determine the area fractions associated with these transitions, we performed Brownian dynamics simulations of $N=2500$ particles, and calculated the local orientational and translational order parameter of each particle $j$, as given by

$$
\psi_{6 j}=\frac{1}{\mathcal{N}_{j}} \sum_{k=1}^{\mathcal{N}_{j}} \mathrm{e}^{6 i \theta_{j k}}
$$

and

$$
\psi_{T j}=\mathrm{e}^{i \mathbf{G} \cdot \mathbf{r}_{j}},
$$

respectively, with $\mathcal{N}_{j}$ the number of nearest neighbours of particle $j$ as determined by a Voronoi construction, $\theta_{j k}$ the angle of the bond between particles $j$ and $k$ relative to an arbitrary reference axis, and $\mathbf{G}$ a primary reciprocal lattice vector. The global order parameters are obtained by averaging over all particles, $\psi_{\alpha}=\frac{1}{N} \sum_{j=1}^{N} \psi_{\alpha j}$, and their susceptibilities are given by $\chi_{\alpha}=\left\langle\left|\psi_{\alpha}{ }^{2}\right|\right\rangle-\left\langle\left|\psi_{\alpha}\right|\right\rangle^{2}$ with $\alpha=6, T$. The orientational susceptibility $\chi_{6}$ and the translational susceptibility $\chi_{\mathrm{T}}$ are plotted in Fig. 1 as a function of the area fraction $\phi$. They display two clear peaks which correspond to the two distinct phase transitions. Based on the position of these peaks, the fluid to hexatic transition occurs at $\phi \approx 0.123$ and the hexatic to crystal transition occurs at $\phi \approx 0.128$. As an extra check, we have also determined the hexatic to crystal transition by renormalizing the Young's modulus (see Appendix). Both methods yield the same transition point within our errorbars.

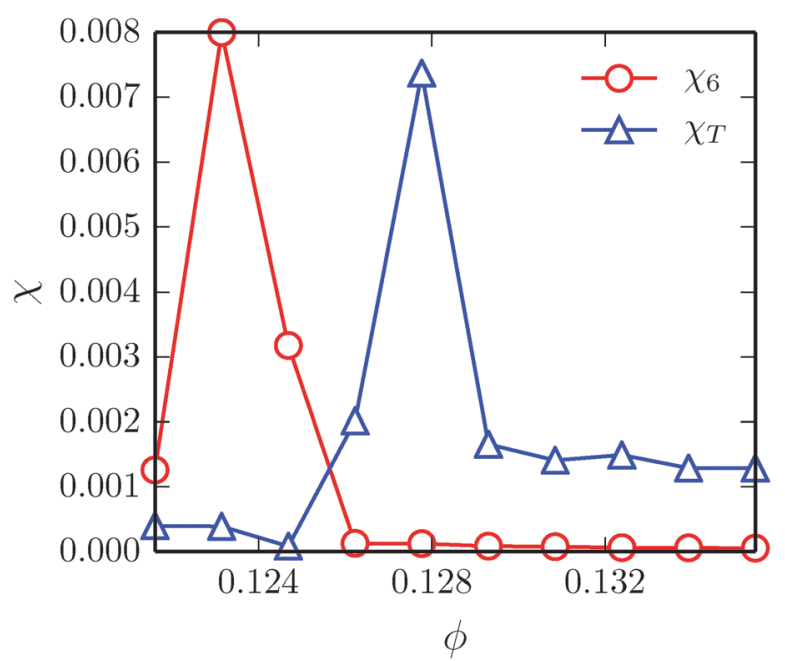

Fig. 1 Susceptibility of the bond orientational order parameter $\chi_{6}$ and the susceptibility of the translational order parameter $\chi_{T}$ as a function of area fraction $\phi$, indicating a fluid to hexatic phase transition at approximately $\phi \approx 0.123$, and a hexatic to crystal transition at approximately $\phi \approx 0.128$.
To investigate the dynamics in this system we use Brownian dynamics simulations consisting of $N=2500$ Yukawa particles, allowing us to reach the long time scales associated with the heterogeneous dynamics. Note that this implies that the system spans 50 unit cells in both directions. For $\phi \approx 0.134$, we have checked that the cooperative behaviour that we observe is robust to variations in system size by running simulations for both smaller systems $N=40^{2}$ as well as larger systems $N=100^{2}$. We define the short-time diffusion time $\tau$ to be the typical time scale on which particles diffuse a distance that is equal to their own dimension $\sigma$, which we determined from measuring the mean square displacement at infinite dilution.

\section{Results}

\section{A. Heterogeneous dynamics}

We start our investigation by examining the dynamic correlation functions of the fluid, hexatic, and crystal phases, similar to the studies of ref. 14 and 15 on (approximately) hard colloids. The mean square displacement for a range of area fractions $\phi$ is shown in Fig. 2(a). From this plot we can identify three different regimes for the single particle dynamics. At short time scales $(t \leq 0.2 \tau)$, the particles diffuse freely and the mean square displacement is density independent and scales with $t$. For the solid and hexatic phases, an intermediate, subdiffusive regime is observed, scaling as $\left\langle\Delta r^{2}(t)\right\rangle \sim t^{b}$ with the subdiffusivity parameter $b<1$. Here the particle dynamics is slowed down due to caging by the other particles. As $\phi$ increases, the intermediate, caging regime becomes longer lived with an even lower subdiffusivity parameter $b$. At long times, the particle motion becomes diffusive again, scaling with $t$. Note that this even happens for densities that correspond to the solid phase, indicating that significant diffusion processes occur.

These three regimes are also apparent in the self-part of the intermediate scattering function

$$
F_{\mathrm{s}}(k, t)=\langle\exp (i \mathbf{k} \cdot[\mathbf{r}(t)-\mathbf{r}(0)])\rangle
$$

with $\mathbf{k}$ the wavevector. In Fig. 2(b) we plot $F_{\mathrm{s}}\left(k_{0}, t\right)$, where $k_{0}=2 \pi / a$ with $a$ the lattice spacing for varying packing fractions $\phi \cdot{ }^{18}$ For solid phases, $F_{\mathrm{s}}\left(k_{0}, t\right)$ shows a clear plateau at intermediate time scales that rises and becomes longer lived with increasing $\phi$. The increase in the height and length of the plateau is indicative of stronger caging.

The intermediate plateaus in the mean square displacement and intermediate scattering function imply that there must be a time scale over which the dynamics is highly heterogeneous. The non-Gaussian parameter

$$
\alpha_{2}(t)=\frac{1}{2} \frac{\left\langle\Delta r^{4}(t)\right\rangle}{\left\langle\Delta r^{2}(t)\right\rangle^{2}}-1
$$

provides a way to quantify the time scale and strength of dynamical heterogeneities. ${ }^{19}$ The non-Gaussian parameter is shown for a range of $\phi$ in Fig. 2(c). For the fluid $\alpha_{2}(t) \approx 0$, while for higher $\phi$ a clear peak in $\alpha_{2}(t)$ is observed. At $t^{*}$, the time for which $\alpha_{2}(t)$ reaches its maximum, the dynamics is most 

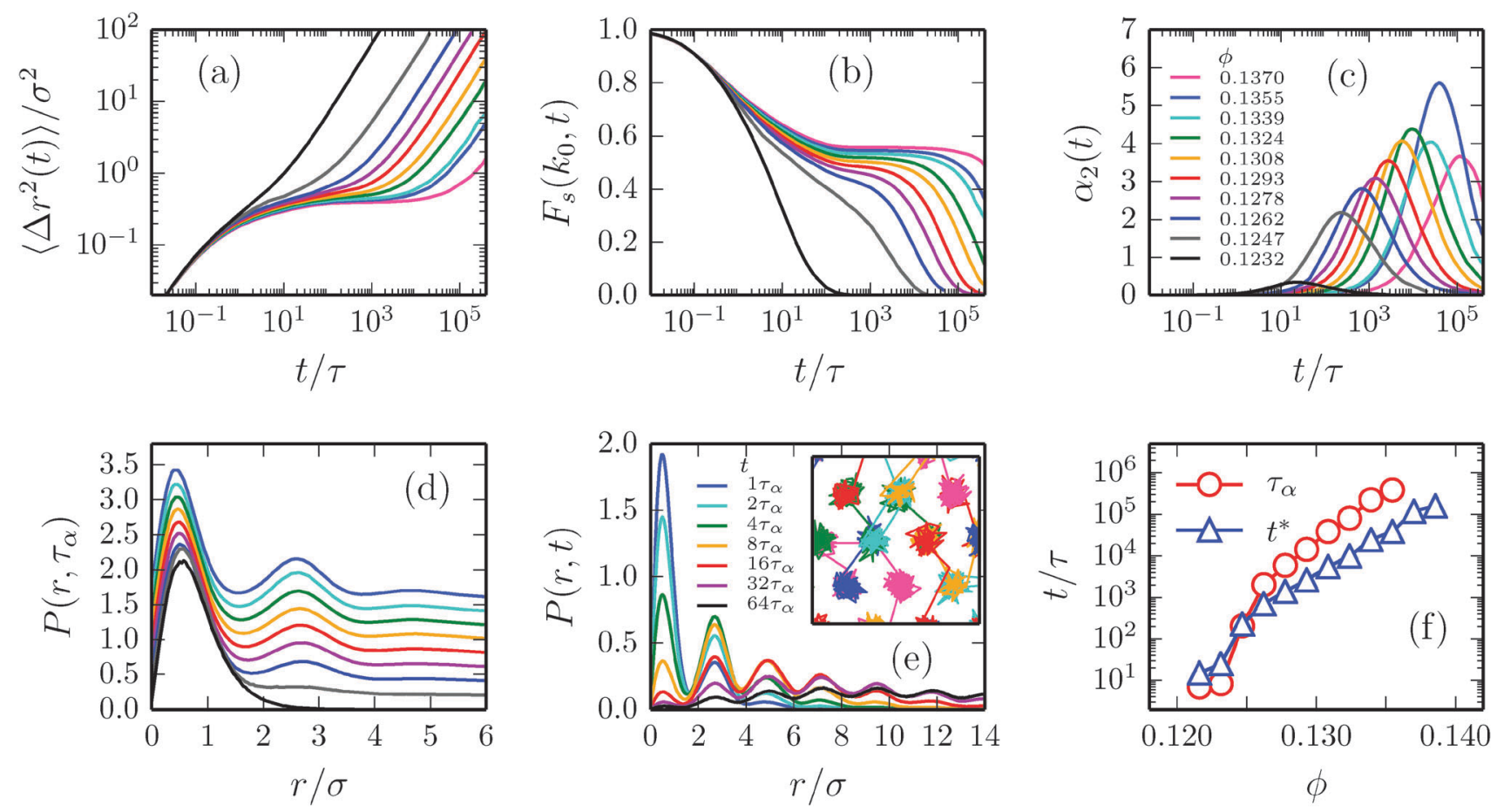

Fig. 2 (a) Mean square displacement $\left\langle\Delta r^{2}(t)\right\rangle$, (b) the self-part of the intermediate scattering function $F_{s}\left(k_{0}, t\right)$, where $k_{0}=2 \pi / a$ with a the lattice spacing (c) non-Gaussian parameter $\alpha_{2}(t)$, (d) and the self-part of the van Hove correlation function $P(r, t)$ at $t=\tau_{\alpha}$. In (d) we shift the otherwise overlapping $P\left(r, \tau_{\alpha}\right)$ curves vertically with steps of 0.2 for sake of clarity. Lines with different colors in (a-d) correspond to different packing fractions $\phi$ as labelled in (c). (e) $P(r, t)$ for $\phi=0.1278$ at different time intervals of integer $\tau_{\alpha}$. In the inset we plot the particle trajectories over $t \approx 4 \tau_{\alpha}$ for a small section of the simulation box, where we use different colors for different particle trajectories. (f) The structural relaxation time $\tau_{\alpha}$ and the time scale of the dynamical heterogeneities $t^{\star}$ in units of the short-time diffusion time $\tau$ as a function of $\phi$.

heterogeneous. The time scale $t^{*}$ of the dynamical heterogeneity increases with $\phi$. Additionally, the magnitude of dynamical heterogeneity $\alpha_{2}\left(t^{*}\right)$ increases with $\phi$ up to $\phi \approx 0.134$. This increase is consistent with what was observed in ref. 14 and 15 . Note that at the highest densities, the dynamics becomes increasingly slow making it hard to sample properly the dynamics at relevant time scales, likely leading to the lowering of the peaks observed above $\phi \approx 0.134$.

To further explore the dynamical heterogeneities, we calculated the displacement histogram $P(r, t) \equiv 2 \pi r G_{\mathrm{s}}(r, t)$, where $G_{\mathrm{s}}(r, t)=\langle\delta(r-|\mathbf{r}(t)-\mathbf{r}(0)|)\rangle$ is the self-part of the van Hove function. In Fig. 2(d), we plot $P\left(r, \tau_{\alpha}\right)$, i.e. the displacement histogram evaluated at the relaxation time $\tau_{\alpha}$, with $\tau_{\alpha}$ defined by $F_{\mathrm{s}}\left(k=k_{0}, t=\tau_{\alpha}\right)=e^{-1}$, see Fig. 2(f) for typical values of $\tau_{\alpha}$. In the fluid phase, $P\left(r, \tau_{\alpha}\right)$ displays a single peak at a characteristic displacement length. However, in the hexatic and crystalline phases, this function develops multiple peaks, corresponding to multiple characteristic hopping distances. The extent of particle hopping depends strongly on $\phi$. In Fig. 2(e), this hopping is further illustrated by plotting $P(r, t)$ for different times $t>\tau_{\alpha}$ at fixed $\phi=0.1278$, corresponding to the hexatic phase. We note that over time, the number of peaks increases. This is evidence of jump-like, intermittent particle motion, as depicted in the inset of Fig. 2(e).

The translational relaxation time $\tau_{\alpha}$ and the time at which the displacements are most heterogeneous $t^{*}$ increase rapidly with area fraction $\phi$ in the solid and hexatic phases as shown in
Fig. 2(f). We note that $\tau_{\alpha}$ grows faster than $t^{*}$ as the system becomes more closely packed. This feature has also been observed in super-cooled liquids ${ }^{20}$ and polymer melts. ${ }^{21}$ Note that in the liquid phase, we do not observe a significant difference between the two time scales, as expected.

\section{B. Microscopic mechanisms for diffusion in crystal and hexatic phases}

As shown in the previous section, particle hopping clearly plays an important role in the dynamics in the hexatic and crystal phases. In this section we examine this phenomenon on the single-particle level. We start by studying time-lapse sequences of the crystal and hexatic phases. In the following we distinguish two separate mechanisms for diffusion: (i) a completely coherent, cooperative rotation of a small number of particles (rearrangement loop), and (ii) the creation, diffusion and annihilation of vacancy-interstitial pairs.

In Fig. 3, we show a time-lapse sequence of a rearrangement loop inside a crystal at area fraction $\phi=0.1339$, and follow the topological evolution of the defect structure accompanying one of these cooperative events by constructing Voronoi diagrams. Starting from a defect-free configuration [Fig. 3(a)], we first observe the spontaneous formation of a ring-shaped grain boundary, corresponding to the alternating 5 - and 7 -fold defects in the Voronoi construction [Fig. 3(b)]. Here a 5- and 7 -fold defect is defined as a particle that possesses 5 and 7 nearest neighbours, respectively. After completing the cooperative 

(a) $\mathrm{t}=0 \tau$
(b) $\mathrm{t}=5.0 \tau$
(c) $\mathrm{t}=10.0 \tau$
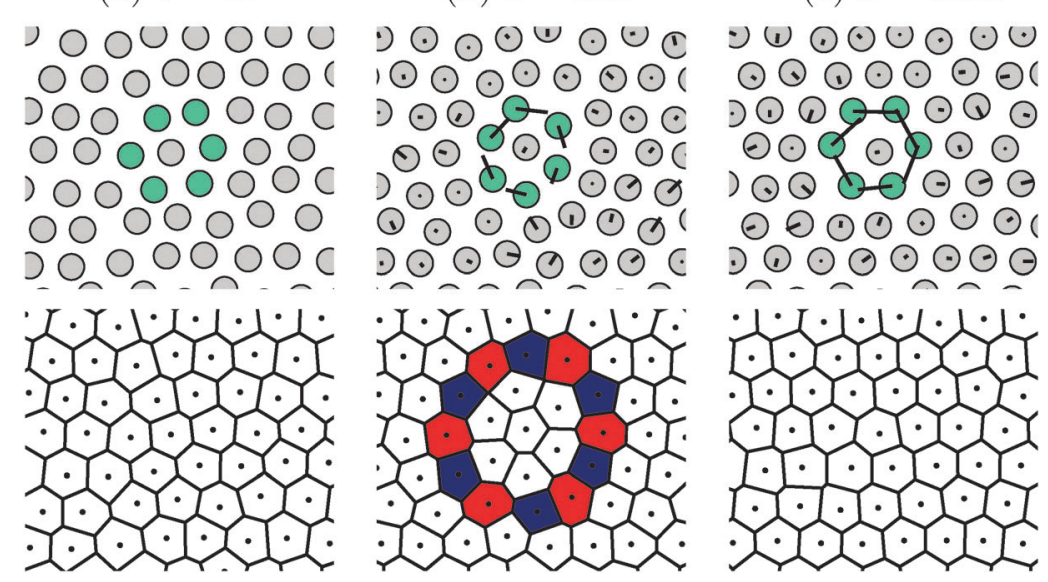

Fig. 3 Time-lapse sequence of simulated configurations (top row) and corresponding Voronoi tessellations (bottom row) showing a spontaneous loop rearrangement (green particles) inside a crystal phase with $\phi=0.1339$. We have superimposed lines that connect the current particle positions with the initial defect-free configuration at $t=0 \tau$ as displayed in (a). In the Voronoi tessellations we color particles with 5 and 7 nearest neighbours by blue and red cells, respectively. The time is indicated above the snapshots with $\tau$ the short-time diffusion time. A large thermal fluctuation causes the formation of a circular grain boundary in which the particles move in a cooperative ring-like fashion (b). The rotational motion correlates with the effective annihilation of the 5- and 7-neighbour defects $(\mathrm{a}-\mathrm{c})$. Once the cooperative rotation is complete, the particles become an integral part of the crystal again (c).

rotational motion, the particles have travelled one integer lattice spacing and become an integral part of the crystal again [Fig. 3(c)]. Interestingly, this motion is somewhat reminiscent of the cooperative motion of sliding rows of particles seen in ref. 22. However, while the motions they observed were system spanning, the ring-like diffusion we observe is only effecting small parts of the system and is not associated with the size of the system we are simulating.

In addition to the closed loops, we observe open-ended strings of cooperative motion associated with vacancy-interstitial pairs, as shown in Fig. 4. During the nucleation of a vacancyinterstitial pair, a region dense in defects forms inside the crystal [Fig. 4(a)]. The dislocations in this locally disordered patch quickly settle into a clear vacancy and interstitial pair, containing each two 5- and 7-neighbour defects. [Fig. 4(b)]. The interstitial and vacancy are connected by a string of displaced particles, which we refer to as a rearrangement string.
To trace the growth of the string we color-code all the string-like moving particles as green, through evaluation of the particle displacements up to the current time, as indicated above the corresponding snapshot. The diffusion of the point defects forms the microscopic mechanism for growth of the string [Fig. 4(c)]; strings grow both on the interstitial-side where particles are somewhat displaced from their equilibrium position to make room for the interstitial, as well as on the vacancy-side, where particles are free to explore the extra free area that was previously occupied by other particles. Eventually, the vacancy and interstitial come into contact with their complement, and annihilate (as shown in the zoom in of Fig. 4(d)).

In agreement with other studies, we find that the migration of both these point defects is accompanied by a switching between different topological configurations. ${ }^{6,23}$ Note that both vacancies and interstitials are known to have many distinct topological configurations with different symmetries. ${ }^{6,24}$ (a) $\mathrm{t}=0 \tau$

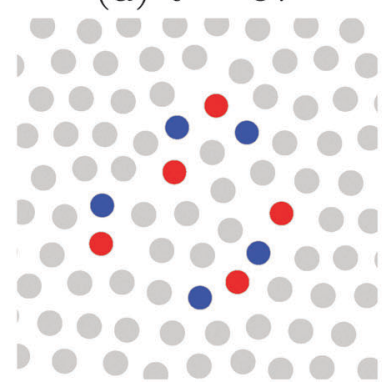

(b) $\mathrm{t}=2.4 \tau$

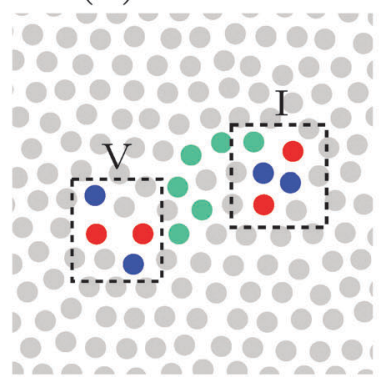

(c) $\mathrm{t}=85.4 \tau$

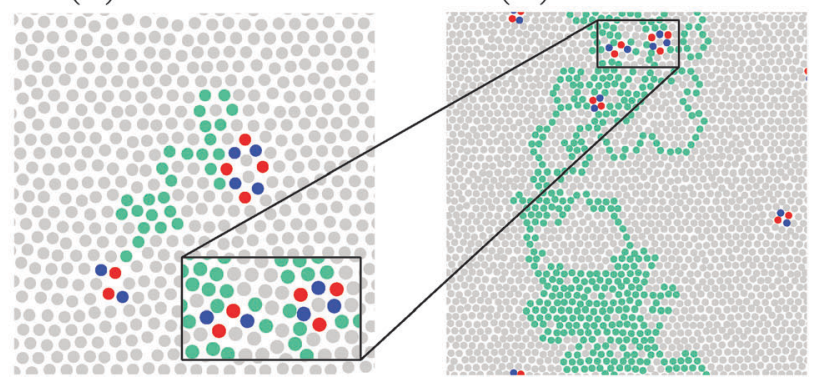

Fig. 4 Time-lapse sequence of simulated configurations showing a spontaneous string of rearrangements (green particles) inside a crystal phase with $\phi=0.137$. The time is indicated above the snapshots with $\tau$ the short-time diffusion time. We color-code particles with 5 or 7 nearest neighbours by blue and red, respectively. (a) A region dense in defects forms inside the crystal due to a large thermal agitation. (b) The defected configuration quickly arranges into a clear vacancy and interstitial, indicated with a $V$ and I, containing both two 5- and two 7-neighbour defects. (c) Many particle rearrangements occur as both the vacancy and interstitial diffuse along the string of rearrangements. (d) The vacancy and interstitial come into proximity again and annihilate in successive frames (zoom). 
In Fig. 4(b) both the vacancy (left defect) and the interstitial (right defect) contain two 5- and 7-neighbour defects and have two-fold symmetry. Note that in examining the migration of these defects we did not observe any topological signatures of di-vacancies or higher order vacancies. This may very well be due to the low concentration of point defects in our system.

In the majority of cases, vacancy-interstitial pairs recombine relatively fast. This fast recombination is driven by the shortrange attraction between a vacancy and an interstitial. ${ }^{25}$ It should be noted that the loops formed by the annihilation of these vacancy-interstitial pairs can be of the same size and shape as the rearrangement loops described previously. However, they appear to proceed through a different topological evolution of the structure of the solid.

We study in more detail the diffusion of vacancies and interstitials associated with open-ended strings by tracking the location of these point defects in time. For a defect containing $M$ particles, the center of mass $\mathbf{r}_{\mathrm{d}}(t)$ is defined as the center of the non-sixfold coordinated colloids, as used in previous studies of point defect dynamics in two-dimensional crystals, ${ }^{5,6}$ i.e.

$$
\mathbf{r}_{\mathrm{d}}(t)=\frac{\sum_{i=1}^{M} \mathbf{r}_{i}(t)\left(1-\delta_{\mathcal{N}_{i}(t), 6}\right)}{\sum_{i=1}^{M}\left(1-\delta_{\mathcal{N}_{i}(t), 6}\right)},
$$

where $\mathcal{N}_{i}(t)$ is the number of nearest neighbours of particle $i$ as determined by Voronoi construction and $\delta_{i, j}$ is the Kronecker delta function. In Fig. 5, we show one sample trajectory $\mathbf{r}_{\mathrm{d}}(t)$ of a vacancy (blue line) and the associated interstitial (red line). As expected, the point defects preferentially travel along the lattice directions. At short time scales the diffusive motion follows an almost one-dimensional path, while at longer time

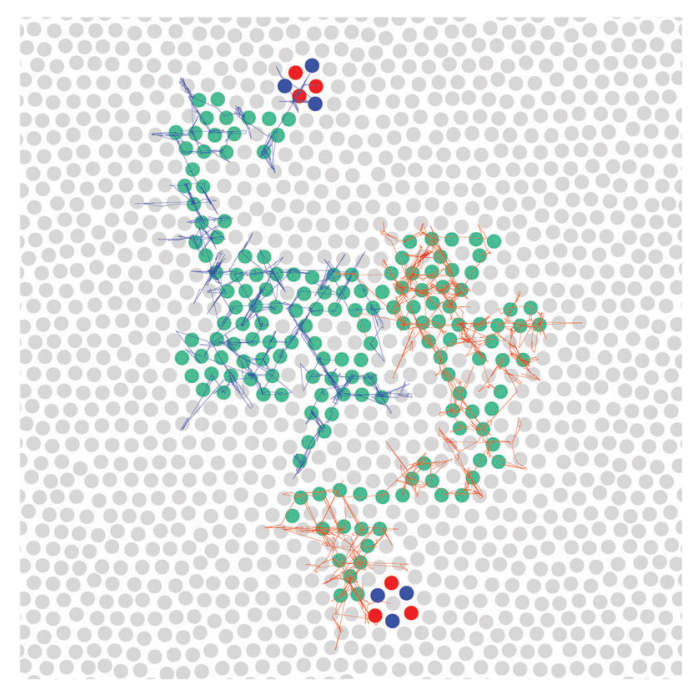

Fig. 5 Trajectories of the vacancy and interstitial correlating strongly with the string-like rearrangements inside a crystal phase with $\phi=0.1339$. Particles that exchanged lattice sites irreversibly are indicated with green. The vacancy and interstitial trajectory are shown in blue and red, respectively. The point defects diffuse along the lattice axis. At short time scales the diffusive motion follows an almost one-dimensional path, while at longer time scales the defect motion is often reoriented along one of the lattice lines.

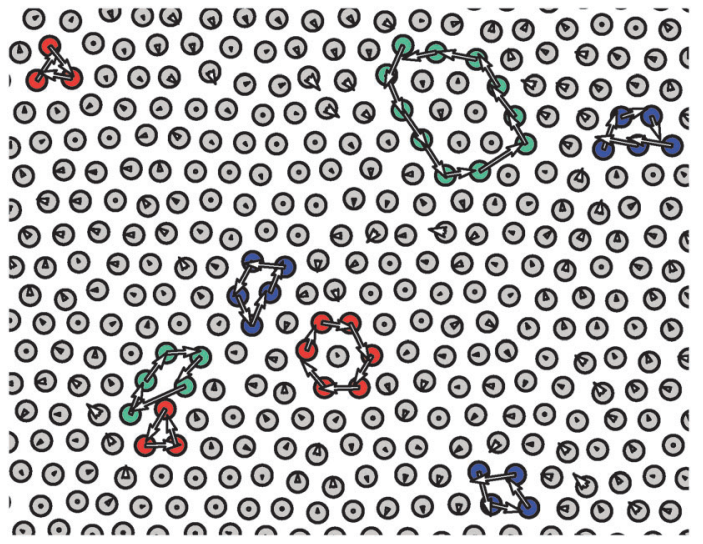

Fig. 6 Simulation snapshot of various types of string-like rearrangements. The superimposed quivers indicate the particle trajectories.

scales the defect motion is often reoriented along one of the lattice lines. ${ }^{5,6}$ The measured trajectories correlate strongly with the positions of particles that have changed lattice site (highlighted in green), indicating that particles are trapped inside their cage until they are untrapped by a diffusing defect.

In our simulations the string-like rearrangements, both openended as well as closed, emerge in a large variety of sizes and geometries, as illustrated in Fig. 6. To investigate these, we identify the cage-breaking particles using a displacement threshold of $\left|\mathbf{r}_{i}(t)-\mathbf{r}_{i}(0)\right|>0.65 a$, which is sufficiently larger than the typical positional fluctuations of particles inside their cages, while at the same time small enough to make sure that we identify all fast particles. Please note that this displacement threshold is approximately equal to the first minimum in the displacement histogram $P(r)$, as shown in Fig. 2(d and e). To determine which particles undergo correlated motion we examine the relative displacement of particles. The mobile particles $i$ and $j$, as identified by the above criterion, are considered in the same rearrangement string when

$$
\min \left[\left|\mathbf{r}_{i}(t)-\mathbf{r}_{j}(0)\right|,\left|\mathbf{r}_{i}(0)-\mathbf{r}_{j}(t)\right|\right]<0.3 a,
$$

with $a$ the lattice spacing. This criterion was used in previous studies of string-like motion. ${ }^{8,26,27}$ As a time interval we use $t=t^{*}$, at which the dynamical correlations reach a maximum, which provides a well-defined interval to monitor the cooperative dynamics. ${ }^{8,28,29}$ In our analysis we consider only strings of size $n \leq 30$, thus minimizing possible finite-size effects where vacancies and interstitials interact with each other through the periodic boundaries of the box.

The distribution of string lengths $P(n)$ depends strongly on the packing fraction $\phi$ as shown in Fig. 7(a). In solid phases all $P(n)$ overlap in the range of $n$ that we consider and exhibits a power law-like decay $P(n) \sim n^{-\mu}$ with exponent $\mu \approx 2.5$. We can show, using a very simple model, that the observed power law-like decay of $P(n)$ for our crystals can be explained by the diffusive motion of defects inside the lattice. To illustrate this we perform a lattice simulation in which we randomly move a vacancy or interstitial on a hexagonal lattice, causing many stringlike rearrangements. This allows us to extract the $P(n)$ associated with a point defect moving according to a random walk. Clearly, 


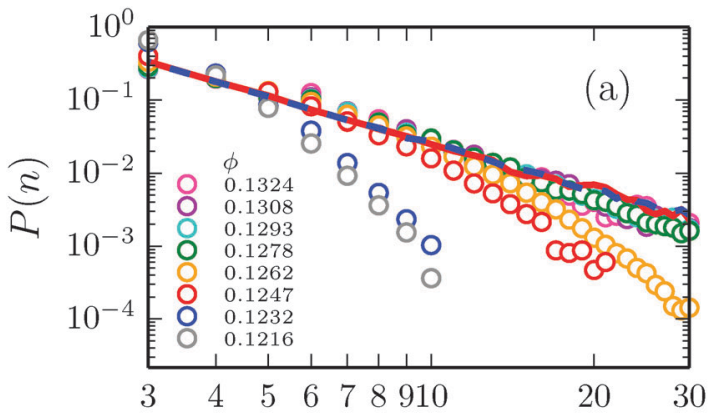

n

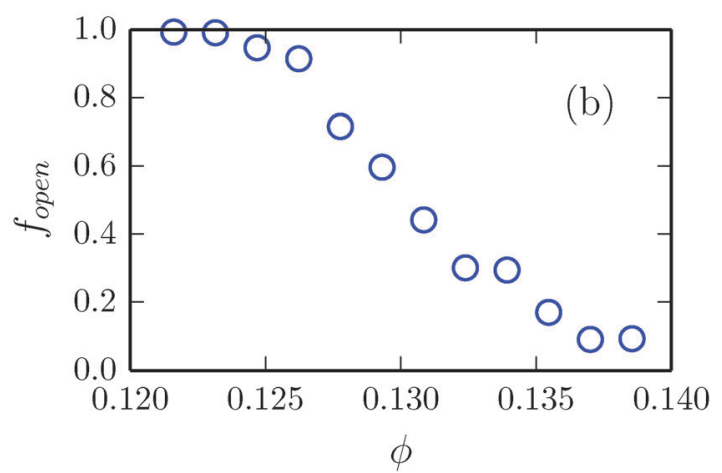

Fig. 7 (a) String size distribution $P(n)$ at several packing fractions $\phi$. The red and blue dashed lines indicate the results for our lattice model of a diffusing interstitial and vacancy, respectively. (b) Fraction of string-like moving particles that are part of an open string $f_{\text {open }} v s$. $\phi$.

the $P(n)$ that we obtain for our model of a diffusing defect on a hexagonal lattice fits our data well, obeying similar power law decay of $P(n) \sim n^{-\mu}$ with exponent $\mu \approx 2.15$, as indicated in Fig. 7(a) with red and blue dashed lines for the diffusing interstitial and vacancy, respectively. These results indicate that the power-law behaviour originates from the fact that defects diffuse on a lattice and have a certain probability of either diffusing to previously unexplored lattice sites or to prior lattice sites; thus occasionally breaking a larger rearrangement string into two smaller, separate rearrangement strings. Note that our lattice model is highly simplified compared to the real system, for example it does not take into account the spontaneous rearrangement loops as shown in Fig. 3 and assumes the point defects to be non-interacting, which may very well explain the small difference in $\mu$.

At area fractions below the solid to hexatic transition, corresponding to densities of $\phi \leq 0.1262$ in Fig. 7(a), the string-like nature of the particle motions becomes increasingly less pronounced, leading to an exponential decay of $P(n)$, indicating that independent particle motion starts to become dominant over cooperative cage breaking [Fig. 7(a)]. Such exponential decay of the string-size distribution has also been observed in supercooled liquids. ${ }^{26}$ Additionally, strings undergo a gradual topological transition from closed loop-like to openended as $\phi$ decreases as shown in Fig. 7(b). We note that such a transition in cooperative motion from closed loop-like to open-ended cooperative motion has also been observed during melting from superheated states in three-dimensional crystalline solids. ${ }^{7,8}$ We propose that this transition originates from two effects. Firstly, in the crystal phase the attraction between vacancies and interstitials is expected to become weaker with decreasing $\phi$ leading to longer times before the two ends of a rearrangement string meet and close. Secondly, in the crystal phase particles move from lattice point to lattice point while in a fluid there is no lattice and particles do not necessarily have to take over the position of their predecessors. Thus there should be little reason for particles to diffuse in a closed configuration.

\section{Conclusions}

In conclusion, we have examined the dynamics in the hexatic and crystalline phases of the two dimensional, soft colloidal system that was experimentally realized in ref. 17. Dynamic correlation functions clearly indicate that the dynamics in both these phases is highly heterogeneous, in agreement with what was observed in ref. 14 and 15 for (approximately) hard colloidal systems. We measured the time scale associated with these heterogeneities and examination of displacement histograms associated with this system indicated that the heterogeneous motion stems from particle hopping. A closer investigation of the single particle dynamics in this system has revealed, in agreement with ref. 17, that the diffusion in the hexatic and crystalline phases arises due to two distinct local particle rearrangements, namely (i) the coherent, and almost simultaneous rearrangement of a loop of particles that leave the local structure of the solid undisturbed, and (ii) the diffusion of vacancy-interstitial pairs. This of course raises the interesting question whether these two mechanisms capture all the essential dynamics associated with the heterogeneous dynamics. In the crystal phase, we can be fairly certain that we have captured all the essential dynamics as the underlying lattice allows us to fully identify all cage-breaking events, and we find that these events can always be categorized in one of the two distinct local particle arrangements identified above. In contrast, for the hexatic phase, there is no underlying lattice and local positional order is expected to decay over sufficiently long time scales. As a result, other forms of motion may be expected to occur in this phase as well.

To further explore the rearrangement strings, we also examined the string-size distributions. For the crystal phase, we found that it exhibits a power law-like decay, which could be approximately matched by a simple, random walk description of vacancies and interstitials on a lattice. We attribute deviations from this model to the interaction between the interstitials and vacancies and the fact that the spontaneous rearrangement loops were not taken into account. In the hexatic phase, the string-size distribution decays exponentially for the string lengths that we consider, which may very well indicate that other types of motions (not string-like) start to become responsible for diffusion as well.

One may be temped to assume that there is some underlying connection between the heterogeneous dynamics of glassforming systems and crystals. However, such a connection is not evident as in a glass there is no order while in a crystal 
particles are sitting on average on a lattice. The same is true for the dynamics of the system: in a glass particles rearrange to new "random" locations while in a crystal the rearrangements follow the lattice, i.e. particles hop from lattice point to lattice point. Interestingly, we find that the string-size distribution in the crystal phase follows a power law-like decay, in contrast to the exponential decay found in super-cooled liquids. ${ }^{26}$

Lastly, this work was originally inspired by the preliminary observation in ref. 17, that both thermal fluctuations and local mechanical fluctuations are relaxed through similar cooperative modes. The results in this paper continue to fully support this conclusion. We note that these stress relaxation pathways are in contrast to how stress relaxation occurs in systems which are subjected to macroscopic, external stresses. These system typically relax their stresses through the formation and motion of dislocations in the lattice. ${ }^{10-13}$

\section{Appendix}

To obtain an additional prediction of the hexatic to crystal phase transition, we renormalize the Young's modulus following procedures previously presented in ref. 30-32. The elasticity of the hexagonal crystal lattice can be completely described by two independent elastic constants, namely the bulk modulus $B$ and the shear modulus $\mu$. The Young's modulus $K$ of the two-dimensional triangular crystal, which is a combination of the bulk modulus $B$ and the shear modulus $\mu$, is calculated using the relation

$$
K=\frac{8}{\sqrt{3} \rho k_{\mathrm{B}} T} \frac{(\lambda+\mu) \mu}{\lambda+2 \mu},
$$

where Lamé's first parameter $\lambda$ is related to the bulk modulus $B=$ $\lambda+\mu .{ }^{33-35}$ The unrenormalized elastic constants and Young modulus are only valid for a perfect, defect-free, triangular lattice; if topological defects such as dislocations are present a renormalization is required. The small fraction of vacancies and interstitials $(\sim 0.0004)$ that we observe in the solid phase is unlikely to alter the elasticity substantially. ${ }^{36,37}$ However, as pointed out in the KTHNY theory the presence of dislocations lowers the elasticity considerably. . $^{34,35,37,38}$

As a first step to renormalise the Young's modulus, we determine the dislocation core energy $E_{\mathrm{c}}$. Assuming that the core radius is given by $r_{\mathrm{c}}=a$, the core energy of a dislocation is directly related to the dislocation probability:

$$
p_{\mathrm{d}}=\frac{16 \sqrt{3} \pi^{2}}{K-8 \pi} I_{0}\left(\frac{K}{8 \pi}\right) \exp \left(\frac{K}{8 \pi}\right) \exp \left(\frac{-2 E_{\mathrm{c}}}{k_{\mathrm{B}} T}\right),
$$

where $p_{\mathrm{d}}$ is the fraction of dislocation pairs per unit cell, and $I_{0}$ and $I_{1}$ are Bessels functions. ${ }^{38,39}$ Note that $p_{\mathrm{d}}=n_{\mathrm{dp}} / N$ with $n_{\mathrm{dp}}$ the number of dislocation pairs and $N$ the number of particles. We renormalize the Young's modulus $K$ and the fugacity of dislocations $y$ using the KTHNY recursion relations ${ }^{34,35}$

$$
\begin{aligned}
\frac{\mathrm{d} K^{-1}(l)}{\mathrm{d} l}= & \frac{3}{4} \pi y^{2}(l) \exp \left(\frac{K(l)}{8 \pi}\right) \\
& \times\left[2 I_{0}\left(\frac{K(l)}{8 \pi}\right)-I_{1}\left(\frac{K(l)}{8 \pi}\right)\right]
\end{aligned}
$$
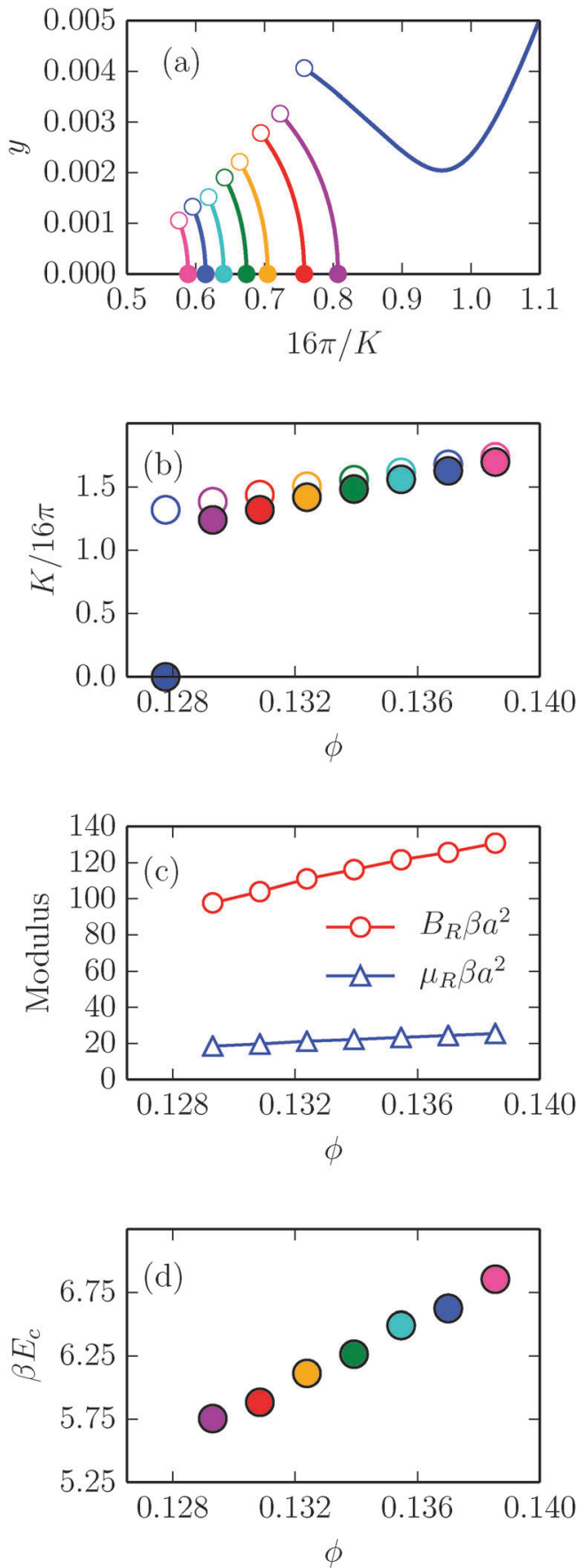

Fig. 8 (a) $y-K$ trajectory upon renormalization for $\phi=0.1385,0.1370$, $0.1355,0.1339,0.1324,0.1308,0.1293,0.1278$ from left to right. (b) Unrenormalized (open markers) and renormalized (solid markers) Young's moduli $K$ vs. $\phi$. (c) The renormalized bulk $B_{\mathrm{R}}$ and shear $\mu_{\mathrm{R}}$ moduli vs. $\phi$. Here we have rendered the elastic moduli dimensionless through multiplication with $a^{2} / k_{\mathrm{B}} T$. (d) Dimensionless dislocation core energy $\beta E_{\mathrm{c}} v s$. $\phi$.

$$
\begin{aligned}
\frac{\mathrm{d} y(l)}{\mathrm{d} l}= & \left(2-\frac{K(l)}{8 \pi}\right) y(l) \\
& +2 \pi y^{2}(l) \exp \left(\frac{K(l)}{16 \pi}\right) I_{0}\left(\frac{K(l)}{8 \pi}\right),
\end{aligned}
$$


with $l$ the coarse-graining length scale, which yield the thermodynamic values of $K$ and $y$ in the limit $l \rightarrow \infty$. The unrenormalized Young's modulus $K(l=0)$ and $y(l=0)=\exp \left(-E_{\mathrm{c}} / k_{\mathrm{B}} T\right)$ serve as the initial conditions of the set of coupled differential equations [eqn (7) and (8)]. The renormalisation recursion relations can now be solved numerically by using a standard Euler forward discretization.

We show the trajectories in the $y-K$ plane for different packing fractions in Fig. 8(a). Clearly, there are two types of trajectories. For $\phi \geq 0.1293$ the fugacity of dislocations $y(l) \rightarrow 0$ for $l \rightarrow \infty$, giving rise to an ordered crystalline phase, while for $\phi \leq 0.1278$ the fugacity of dislocations $y(l) \rightarrow \infty$ for $l \rightarrow \infty$, giving rise to a disordered hexatic phase. According to the KTHNY theory the abrupt jump in $K_{\mathrm{R}}$ from $16 \pi$ to 0 is driven by the proliferation of free dislocations, i.e. the formation of pairs of particles having five and seven nearest neighbours.

We plot the renormalized Young's modulus $K_{\mathrm{R}}$ in Fig. 8(b) and the dimensionless, renormalized bulk $B_{\mathrm{R}}$ and shear $\mu_{\mathrm{R}}$ moduli in Fig. 8(c). The renormalized Young's modulus $K_{\mathrm{R}}$ decreases as the packing fraction $\phi$ decreases. The decrease in the elasticity of the lattice implies that cages become weaker and that the energy barrier for particle hopping is reduced. In these weak crystal lattices, large thermal excitations are frequently relaxed through cooperative decaging motions of particles by means of the formation and migration of defects like vacancies and interstitials.

The dimensionless dislocation core energy at the transition point $\beta E_{\mathrm{c}}{ }^{*} \approx 5.8$ exceeds $2.84 k_{\mathrm{B}} T$, indicating that the solid will melt into a hexatic phase through the unbinding of dislocation pairs. ${ }^{40,41}$ We note that $\beta E_{\mathrm{c}}{ }^{*}$ is in good agreement with experiments on two-dimensional charged colloidal crystals. ${ }^{42}$

\section{Acknowledgements}

We acknowledge financial support from the Dutch Sector Plan Physics and Chemistry, and the Netherlands Organization for Scientific Research (NWO-VENI grant No. 680.47.432). We would also like to thank Frank Smallenburg for many useful discussions.

\section{References}

1 H. Huntington and F. Seitz, Phys. Rev., 1942, 61, 315.

2 C. Zener, Acta Crystallogr., 1950, 3, 346.

3 H. Huntington, Phys. Rev., 1953, 91, 1092.

4 S. A. Rice, Phys. Rev., 1958, 112, 804.

5 A. Pertsinidis and X. Ling, Nature, 2001, 413, 147.

6 A. Libal, C. Reichhardt and C. O. Reichhardt, Phys. Rev. E: Stat., Nonlinear, Soft Matter Phys., 2007, 75, 011403.

7 X.-M. Bai and M. Li, Phys. Rev. B: Condens. Matter Mater. Phys., 2008, 77, 134109.

8 H. Zhang, M. Khalkhali, Q. Liu and J. F. Douglas, J. Chem. Phys., 2013, 138, 12A538.

9 Z. Wang, et al., Science, 2012, 338, 87.
10 G. I. Taylor, Proc. R. Soc. London, Ser. A, 1934, 145, 362.

11 M. Polanyi, Z. Phys., 1934, 89, 660.

12 E. Orowan, Z. Phys., 1934, 89, 605.

13 J. P. Hirth and J. Lothe, Theory of dislocations, Wiley, New York, 1982.

14 R. Zangi and S. A. Rice, Phys. Rev. Lett., 2004, 92, 035502.

15 J. Kim, C. Kim and B. J. Sung, Phys. Rev. Lett., 2013, 110, 047801.

16 H. Shiba, A. Onuki and T. Araki, Europhys. Lett., 2009, 86, 66004 .

17 B. van der Meer, et al., Proc. Natl. Acad. Sci. U. S. A., 2014, 111, 15356.

18 The lattice spacing $a$ was approximated by assuming a hexagonal lattice, and choosing the lattice spacing from the imposed density.

19 A. Rahman, Phys. Rev., 1964, 136, A405.

20 M. D. Ediger, Annu. Rev. Phys. Chem., 2000, 51, 99.

21 F. W. Starr, J. F. Douglas and S. Sastry, J. Chem. Phys., 2013, 138, $12 \mathrm{~A} 541$.

22 B. Alder, W. Hoover and T. Wainwright, Phys. Rev. Lett., 1963, 11, 241.

23 L. DaSilva, L. Candido, G.-Q. Hai and O. Oliveira Jr, Appl. Phys. Lett., 2011, 99, 031904.

24 A. Pertsinidis and X. Ling, Phys. Rev. Lett., 2001, 87, 098303.

25 S. Kim, et al., Proc. SPIE, 2011, 80970X.

26 C. Donati, et al., Phys. Rev. Lett., 1998, 80, 2338.

27 H. Zhang, P. Kalvapalle and J. F. Douglas, Soft Matter, 2010, 6, 5944 .

28 C. Donati, et al., Phys. Rev. E: Stat. Phys., Plasmas, Fluids, Relat. Interdiscip. Top., 1999, 60, 3107.

29 A. Patti, D. El Masri, R. van Roij and M. Dijkstra, Phys. Rev. Lett., 2009, 103, 248304.

30 J. R. Ray, Comput. Phys. Rep., 1988, 8, 109.

31 J. Lutsko, J. Appl. Phys., 1989, 65, 2991.

32 E. Voyiatzis, Comput. Phys. Commun., 2013, 184, 27.

33 J. M. Kosterlitz and D. J. Thouless, J. Phys. C: Solid State Phys., 1973, 6, 1181.

34 D. R. Nelson and B. Halperin, Phys. Rev. B: Condens. Matter Mater. Phys., 1979, 19, 2457.

35 A. Young, Phys. Rev. B: Condens. Matter Mater. Phys., 1979, $19,1855$.

36 M. A. Bates and D. Frenkel, Phys. Rev. E: Stat. Phys., Plasmas, Fluids, Relat. Interdiscip. Top., 2000, 61, 5223.

37 S. Sengupta, P. Nielaba, M. Rao and K. Binder, Phys. Rev. E: Stat. Phys., Plasmas, Fluids, Relat. Interdiscip. Top., 2000, 61, 1072.

38 S. Sengupta, P. Nielaba and K. Binder, Phys. Rev. E: Stat. Phys., Plasmas, Fluids, Relat. Interdiscip. Top., 2000, 61, 6294.

39 D. S. Fisher, B. Halperin and R. Morf, Phys. Rev. B: Condens. Matter Mater. Phys., 1979, 20, 4692.

40 K. J. Strandburg, Phys. Rev. B: Condens. Matter Mater. Phys., 1986, 34, 3536.

41 S. T. Chui, Phys. Rev. B: Condens. Matter Mater. Phys., 1983, 28, 178.

42 C. A. Murray and D. H. Van Winkle, Phys. Rev. Lett., 1987, 58, 1200. 